PROCEEDINGS OF THE

AMERICAN MATHEMATICAL SOCIETY

Volume 130, Number 9, Pages 2543-2551

S 0002-9939(02)06346-3

Article electronically published on March 12, 2002

\title{
PEIRCE GRADINGS OF JORDAN SYSTEMS
}

\author{
JOSÉ A. ANQUELA AND TERESA CORTÉS \\ (Communicated by Lance W. Small) \\ Dedicated to the memory of Eulalia García Rus
}

\begin{abstract}
In this paper we prove that the diagonal components $V_{0}$ and $V_{2}$ of a Peirce grading of a Jordan pair or triple system $V$, inherit strong primeness, primitivity and simplicity from $V$.
\end{abstract}

\section{INTRODUCTION}

Peirce gradings $V=V_{2} \oplus V_{1} \oplus V_{0}$ of a Jordan pair $V$ are introduced in [8, 11] as a generalization of Peirce decompositions with respect to idempotents. In [8] 4.1] it is shown that the components $V_{\alpha}, \alpha=0,1,2$, inherit nondegeneracy and regularity, among other conditions.

In the first section of this paper we show that $V_{0}$ and $V_{2}$ inherit strong primeness, primitivity, semiprimitivity and simplicity from $V$. The proof follows from the corresponding results on inheritance of regularity conditions by subquotients [3, 5 . 6] 8), once it is shown that $V_{0}$ and $V_{2}$ are isomorphic to certain subquotients of $V$.

In the second section we extend the above results to Peirce gradings of triple systems. Dealing with arbitrary Peirce gradings (instead of Peirce decompositions related to idempotents) has a cost, namely the existence of ideals which are not graded. This difficulty is overcome by introducing the notion of graded tight double pair which is used to link Jordan triple systems and Jordan pairs, both with a Peirce grading. The properties of graded tight double pairs established in the second section of the paper are based on a study of ideals in Jordan pairs with a Peirce grading done in the previous section.

Since Peirce decompositions $T=T_{2}(e) \oplus T_{1}(e) \oplus T_{0}(e)$ of a Jordan triple system $T$ with respect to a tripotent $e \in T$ are particular cases of Peirce gradings, the main result of the second section contains as a corollary the central result of McCrimmon [9] on the simplicity of $T_{0}(e)$ and $T_{2}(e)$ when $T$ is simple. However, the proof given in this paper cannot be considered an alternative to the proof by McCrimmon in 9]. Indeed the results of this paper are based on the results of [5], which depend on the inheritance of regularity by local algebras proved in [6]. These latter results are based on the results by McCrimmon [9].

Received by the editors June 16, 2000 and, in revised form, April 16, 2001.

2000 Mathematics Subject Classification. Primary 17C27, 17C10, 17C20.

Key words and phrases. Jordan system, Peirce grading, simple, primitive, strongly prime.

This work was partially supported by the DGES, PB97-1069-C02-02 and the Ministerio de Ciencia y Tecnología, BFM2001-1938-C02-02. 


\section{Preliminaries}

0.1. We will deal with Jordan pairs and triple systems over an arbitrary ring of scalars $\Phi$. The reader is referred to [3, 4, 7, 10] for basic results, notation and terminology, though we will stress some definitions and basic notions.

- For a Jordan pair $V=\left(V^{+}, V^{-}\right)$we will denote the products by $Q_{x} y$, for any $x \in V^{\sigma}, y \in V^{-\sigma}, \sigma= \pm$, with linearizations denoted by $Q_{x, z} y=\{x, y, z\}$.

-A Jordan triple system $T$ is given by its products $P_{x} y$, for any $x, y \in T$, with linearizations denoted by $P_{x, z} y=\{x, y, z\}$.

0.2. By doubling any Jordan triple system $T$ one obtains the double Jordan pair $V(T)=(T, T)$ with products $Q_{x} y=P_{x} y$, for any $x, y \in T$. From a Jordan pair $V=\left(V^{+}, V^{-}\right)$one can get a (polarized) Jordan triple system $T(V)=V^{+} \oplus V^{-}$by defining $P_{x^{+} \oplus x^{-}}\left(y^{+} \oplus y^{-}\right)=Q_{x^{+}} y^{-} \oplus Q_{x^{-}} y^{+}$[7, 1.13, 1.14].

\subsection{Regularity conditions through the functors $V$ and $T$ :}

(i) For a Jordan triple system $T$, we have:

(a) $T$ is nondegenerate if and only if $V(T)$ is nondegenerate,

(b) if $V(T)$ is strongly prime, then $T$ is strongly prime,

(c) if $V(T)$ is simple, then $T$ is simple.

(ii) For a Jordan pair $V$, we have:

(a) $V$ is nondegenerate if and only if $T(V)$ is nondegenerate,

(b) $V$ is strongly prime if and only if $T(V)$ is strongly prime,

(c) $V$ is simple if and only if $T(V)$ is simple.

(i)(a) and (ii)(a) are straightforward; (i)(b)(c) follow from the fact that any nonzero ideal $I$ of $T$ gives rise to the nonzero ideal $V(I)=(I, I)$ of $V(T)$; (ii)(b)(c) are proved in [1, pg. 230].

0.4. Let $V$ be a Jordan pair, and let $M \subseteq V^{+}$be an inner ideal of $V$. The set

$$
\operatorname{Ker}_{V} M=\operatorname{Ker} M=\left\{x \in V^{-} \mid Q_{M} x=Q_{M} Q_{x} M=0\right\}
$$

gives rise to the ideal $(0, \operatorname{Ker} M)$ of the subpair $\left(M, V^{-}\right)$of $V$. The quotient

$$
\left(M, V^{-}\right) /(0, \operatorname{Ker} M)=\left(M, V^{-} / \operatorname{Ker} M\right)
$$

is called the subquotient of $V$ determined by $M$. Similarly, one can consider subquotients of $V$ determined by an inner ideal $M \subseteq V^{-}$of $V$ (see [8]).

If $V$ is nondegenerate, then $\operatorname{Ker} M=\left\{x \in V^{-} \mid Q_{M} x=0\right\}$ [8, 2.4].

0.5. Most regularity conditions are inherited by subquotients: Let $V$ be a Jordan pair, $0 \neq M \subseteq V^{\sigma}$ be an inner ideal of $V$, and $S$ be the subquotient of $V$ determined by $M$.

(i) If $V$ is nondegenerate (resp. semiprimitive), then $S$ is nondegenerate (resp. semiprimitive) [8, 2.2].

(ii) If $V$ is strongly prime, then $S$ is strongly prime [6, 3.2(v)].

(iii) If $V$ is primitive, then $S$ is primitive [3, 3.10], [4, 2.2].

Simplicity is not inherited by arbitrary subquotients (cf. [5, 5.2]). However, information about the heart Heart $S$ of $S$ can be obtained when $V$ is simple:

(iv) If $V$ is simple, then $H=$ Heart $S$ of $S$ is simple. Moreover $H^{\sigma}=Q_{M} V^{-\sigma}=$ $Q_{S^{\sigma}} S^{-\sigma}$ and $H^{-\sigma}=S^{-\sigma}[5,5.1]$. 


\section{PEIRCE GRADINGS OF JORDAN PAIRS}

1.1. Following [8, 3.6, 4.1], given a Jordan pair $V=\left(V^{+}, V^{-}\right)$, a Peirce grading of $V$ is a decomposition $V^{\sigma}=V_{2}^{\sigma} \oplus V_{1}^{\sigma} \oplus V_{0}^{\sigma}, \sigma= \pm$ (direct sum of $\Phi$-modules), such that, for any $\alpha, \beta, \gamma \in\{0,1,2\}$,

(i) $Q_{V_{\alpha}^{\sigma}} V_{\beta}^{-\sigma} \subseteq V_{2 \alpha-\beta}^{\sigma}$,

(ii) $\left\{V_{\alpha}^{\sigma}, V_{\beta}^{-\sigma}, V_{\gamma}^{\sigma}\right\} \subseteq V_{\alpha-\beta+\gamma}^{\sigma}$,

(iii) $\left\{V_{0}^{\sigma}, V_{2}^{-\sigma}, V^{\sigma}\right\}=\left\{V_{2}^{\sigma}, V_{0}^{-\sigma}, V^{\sigma}\right\}=0$,

with the convention that $V_{\lambda}^{\sigma}=0$ if $\lambda \notin\{0,1,2\}$. The pairs $\left(V_{\alpha}^{+}, V_{\alpha}^{-}\right)$for $\alpha \in$ $\{0,1,2\}$ will be denoted $V_{\alpha}$, so that $V=V_{2} \oplus V_{1} \oplus V_{0}$.

Notice that the roles of $V_{0}$ and $V_{2}$ can be exchanged in the above definition, i.e., by taking $\tilde{V}_{\alpha}^{\sigma}=V_{2-\alpha}^{\sigma}$, we obtain a new Peirce grading $V=\tilde{V}_{2} \oplus \tilde{V}_{1} \oplus \tilde{V}_{0}$.

Given an idempotent $e$ in a Jordan pair $V$ the usual Peirce decomposition of $V=$ $V_{2}(e) \oplus V_{1}(e) \oplus V_{0}(e)$ with respect to $e$ [7], Section I.5] is an example of Peirce grading of $V$. We remark on the lack of " $(0,2)$-symmetry" in Peirce decompositions given by idempotents, so that the studies of $V_{2}(e)$ and $V_{0}(e)$ require different arguments (cf. [7, 9]).

1.2. From the multiplication rules given in the above definition it is clear that $V_{\alpha}$ is a subpair of $V$ for $\alpha=0,1,2$ and, moreover, $V_{0}^{\sigma}, V_{2}^{\sigma}$ are inner ideals of $V$ whose subquotients will be deeply related to the pairs $V_{0}, V_{2}$, respectively. This relationship, on which the results of this paper are based, is obtained in the following lemma due to Ottmar Loos.

1.3. Lemma (O. Loos). Let $V=V_{2} \oplus V_{1} \oplus V_{0}$ be a Peirce grading of a nondegenerate Jordan pair $V$. The subquotient $S$ of $V$ determined by the inner ideal $M=V_{\alpha}^{\sigma}$ $(\sigma= \pm, \alpha=0,2)$ is isomorphic to $V_{\alpha}$. Indeed, an isomorphism $\varphi: V_{\alpha} \longrightarrow S$ is given by $\varphi^{\sigma}=I d_{V_{\alpha}^{\sigma}}, \varphi^{-\sigma}(x)=x+\operatorname{Ker} M$, for any $x \in V_{\alpha}^{-\sigma}$.

Proof. By (0,2)-symmetry and $(+,-)$-symmetry, we can take $M=V_{2}^{+}$. We will prove the equality $\operatorname{Ker}_{V} M=V_{1}^{-}+V_{0}^{-}$from which everything else readily follows.

Notice that $\operatorname{Ker}_{V} M=\left\{x \in V^{-} \mid Q_{M} x=0\right\}$ by nondegeneracy of $V$, so that $V_{1}^{-}+V_{0}^{-} \subseteq \operatorname{Ker}_{V} M$ by (1.1)(i). Conversely, let $x \in \operatorname{Ker}_{V} M$ and write $x=$ $x_{0}+x_{1}+x_{2}$ with $x_{i} \in V_{i}^{-}(i=0,1,2)$. We just need to show that $x_{2}=0$. Indeed $x_{2} \in \operatorname{Ker}_{V} M$ since $V_{1}^{-}+V_{0}^{-} \subseteq \operatorname{Ker}_{V} M$, hence $x_{2} \in \operatorname{Ker}_{V} M \cap V_{2}^{-}=\operatorname{Ker}_{V_{2}} V_{2}^{+}=0$ by [8, 1.4] since $V_{2}$ is nondegenerate by [8, 4.1(ii)].

Our main result for Peirce gradings of pairs follows from the application of the previous lemma to known results of inheritance of regularity by subquotients (0.5). However, this is not the case for the inheritance of nondegeneracy by Peirce components [8, 4.1(ii)]. That is indeed our starting point since it is needed in the proof of (1.3).

1.4. Theorem. Let $V=V_{2} \oplus V_{1} \oplus V_{0}$ be a Peirce grading of a Jordan pair $V$. Let $\alpha \in\{0,2\}$ and assume $V_{\alpha} \neq 0$. Then:

(i) If $V$ is strongly prime, then $V_{\alpha}$ is strongly prime.

(ii) If $V$ is primitive (resp. semiprimitive), then $V_{\alpha}$ is primitive (resp. semiprimitive).

(iii) If $V$ is simple, then $V_{\alpha}$ is simple. 
Proof. In all cases $V$ is nondegenerate (strongly prime $\equiv$ prime + nondegenerate, primitive $\Longrightarrow$ strongly prime [3, 3.9], semiprimitive $\Longrightarrow$ nondegenerate [7, 4.15], simple $\Longrightarrow$ nondegenerate [5, 2.4 (ii)]), thus $V_{\alpha}$ is nondegenerate by [8, 4.1(ii)], and both $V_{\alpha}^{+}$and $V_{\alpha}^{-}$are nonzero. Moreover, (1.3) applies, so that $V_{\alpha}$ is isomorphic to the subquotient $S$ determined by $V_{\alpha}^{+}$.

(i) follows from (0.5) (ii).

(ii) follows from (0.5) (iii) (resp. (0.5) (i)).

(iii) If $V$ is simple, then the heart of $S$ is simple by (0.5) (iv), hence the heart $H$ of $V_{\alpha}$ is simple. Moreover, using the isomorphism $\varphi: V_{\alpha} \longrightarrow S$ given in (1.3), $H^{-}=\varphi^{-1}\left((\text { Heart } S)^{-}\right)=\varphi^{-1}\left(S^{-}\right)=V_{\alpha}^{-}$. By $(+,-)$-symmetry, also $H^{+}=V_{\alpha}^{+}$, and we have shown $H=V_{\alpha}$, hence $V_{\alpha}$ is simple.

1.5. One of the difficulties when dealing with arbitrary Peirce gradings is the existence of ideals which are not graded. Let us consider an arbitrary grading $V=V_{2} \oplus V_{1} \oplus V_{0}$ of a Jordan pair $V$. An ideal $I$ of $V$ is said to be graded if $I=\left(I \cap V_{2}\right)+\left(I \cap V_{1}\right)+\left(I \cap V_{0}\right)$. Equivalently, if $\pi_{\alpha}: V \longrightarrow V_{\alpha}$ denote the natural projections, $\pi_{\alpha}(I) \subseteq I$ for all $\alpha=0,1,2$. When the Peirce grading considered is a Peirce decomposition with respect to an idempotent $e$ of $V$, every ideal is graded since the projections $\pi_{\alpha}$ are built out of elements of the multiplication algebra of $V$ (cf. 7, Section I.5]).

In general, if $I$ is graded with respect to a Peirce grading $V=V_{2} \oplus V_{1} \oplus V_{0}$, then a Peirce grading is induced in the quotient $V / I$. Namely $V / I=\left(V_{2}+I\right) / I \oplus$ $\left(V_{1}+I\right) / I \oplus\left(V_{0}+I\right) / I$.

To extend (1.4) to triple systems we will need to construct pairs $V$ associated to a triple system $T$ (quotients of $V(T)$ ) which, on the one hand, are tight in the sense that they do not have essentially more ideals than those coming from ideals of $T$, and, on the other hand, behave nicely with respect to a given Peirce grading of $T$. The next result is aimed to find suitable graded ideals of $V(T)$ which will be used to test the behaviour of such $V$.

1.6. Lemma. If $V=V_{2} \oplus V_{1} \oplus V_{0}$ is a Peirce grading of a Jordan pair $V$ and $I$ is an ideal of $V$, then the ideal $M=Q_{I} I=\left(Q_{I^{+}} I^{-}, Q_{I^{-}} I^{+}\right)$of $I$ satisfies $M \subseteq\left(I \cap V_{2}\right)+\left(I \cap V_{1}\right)+\left(I \cap V_{0}\right)$. As a consequence, if $V$ is semiprime, then every nonzero ideal $I$ of $V$ contains a nonzero graded ideal of $V$.

Proof. Let $x \in I^{\sigma}, y \in I^{-\sigma}$. We can write $x=x_{2}+x_{1}+x_{0}, y=y_{2}+y_{1}+y_{0}$, where $x_{\alpha}=\pi_{\alpha}(x), y_{\alpha}=\pi_{\alpha}(y), \alpha=0,1,2$. By the Peirce multiplication rules (1.1),

$$
\begin{gathered}
\pi_{0}\left(Q_{x} y\right)=Q_{x_{0}} y_{0}+Q_{x_{1}} y_{2}+\left\{x_{0}, y_{1}, x_{1}\right\} \\
=\left(Q_{x_{0}} y-Q_{x_{0}}\left(y_{1}+y_{2}\right)\right)+\left(Q_{x} y_{2}-Q_{x_{0}+x_{2}} y_{2}-\left\{x_{0}+x_{2}, y_{2}, x_{1}\right\}\right) \\
+\left(\left\{x_{0}, y_{1}, x\right\}-\left\{x_{0}, y_{1}, x_{0}+x_{2}\right\}\right) \\
=\left(Q_{x_{0}} y-Q_{x_{0}} y_{1}-Q_{x_{0}} y_{2}\right) \\
+\left(Q_{x} y_{2}-Q_{x_{0}} y_{2}-Q_{x_{2}} y_{2}-\left\{x_{0}, y_{2}, x_{2}\right\}-\left\{x_{0}, y_{2}, x_{1}\right\}-\left\{x_{2}, y_{2}, x_{1}\right\}\right) \\
+\left(\left\{x_{0}, y_{1}, x\right\}-\left\{x_{0}, y_{1}, x_{0}\right\}-\left\{x_{0}, y_{1}, x_{2}\right\}\right) \\
={ }_{(\text {by }(1.1))} Q_{x_{0}} y+Q_{x} y_{2}-Q_{x_{2}} y_{2}-\left\{x_{2}, y_{2}, x_{1}\right\}+\left\{x_{0}, y_{1}, x\right\}-\left\{x_{0}, y_{1}, x_{2}\right\} \\
=Q_{x_{0}} y+Q_{x} y_{2}-Q_{x_{2}} y_{2}-\left(\left\{x_{2}, y_{2}, x\right\}-\left\{x_{2}, y_{2}, x_{0}\right\}-\left\{x_{2}, y_{2}, x_{2}\right\}\right)
\end{gathered}
$$




$$
\begin{gathered}
+\left\{x_{0}, y_{1}, x\right\}-\left(\left\{x_{0}, y, x_{2}\right\}-\left\{x_{0}, y_{0}, x_{2}\right\}-\left\{x_{0}, y_{2}, x_{2}\right\}\right) \\
={ }_{(\operatorname{by}(1.1))} Q_{x_{0}} y+Q_{x} y_{2}-Q_{x_{2}} y_{2}-\left\{x_{2}, y_{2}, x\right\}+2 Q_{x_{2}} y_{2}+\left\{x_{0}, y_{1}, x\right\}-\left\{x_{0}, y, x_{2}\right\} \\
=Q_{x_{0}} y+Q_{x} y_{2}-\left\{x_{2}, y_{2}, x\right\}+\left(Q_{x_{2}} y-Q_{x_{2}} y_{0}-Q_{x_{2}} y_{1}\right)+\left\{x_{0}, y_{1}, x\right\}-\left\{x_{0}, y, x_{2}\right\} \\
={ }_{(\operatorname{by}(1.1))} Q_{x_{0}} y+Q_{x} y_{2}-\left\{x_{2}, y_{2}, x\right\}+Q_{x_{2}} y+\left\{x_{0}, y_{1}, x\right\}-\left\{x_{0}, y, x_{2}\right\} \in I^{\sigma}
\end{gathered}
$$

since $x \in I^{\sigma}$ and $y \in I^{-\sigma}$.

We have shown $\pi_{0}\left(M^{\sigma}\right) \subseteq I^{\sigma}, \sigma= \pm$, i.e., $\pi_{0}(M) \subseteq I$. By $(0,2)$-symmetry, $\pi_{2}(M) \subseteq I$ and, hence, $\pi_{1}(M) \subseteq I$ : for any $m \in M^{\sigma}, \pi_{1}(m)=m-\pi_{0}(m)-$ $\pi_{2}(m) \in M+I \subseteq I$ since $M \subseteq I$. Therefore, $M \subseteq \pi_{2}(M)+\pi_{1}(M)+\pi_{0}(M) \subseteq$ $\left(I \cap V_{2}\right)+\left(I \cap V_{1}\right)+\left(I \cap V_{0}\right)$.

If $V$ is semiprime and $I \neq 0$, then $M \neq 0$, hence $\left(I \cap V_{2}\right)+\left(I \cap V_{1}\right)+\left(I \cap V_{0}\right) \neq 0$ and the ideal of $V$ generated by $\left(I \cap V_{2}\right)+\left(I \cap V_{1}\right)+\left(I \cap V_{0}\right)$ is a nonzero graded ideal of $V$ contained in $I$.

\section{Peirce Gradings of Jordan triple Systems}

2.1. Given a Jordan triple system $T$, a Peirce grading of $T$ is a decomposition $T=T_{2} \oplus T_{1} \oplus T_{0}$ (direct sum of $\Phi$-modules) such that, for any $\alpha, \beta, \gamma \in\{0,1,2\}$,

(i) $P_{T_{\alpha}} T_{\beta} \subseteq T_{2 \alpha-\beta}$,

(ii) $\left\{T_{\alpha}, T_{\beta}, T_{\gamma}\right\} \subseteq T_{\alpha-\beta+\gamma}$,

(iii) $\left\{T_{0}, T_{2}, T\right\}=\left\{T_{2}, T_{0}, T\right\}=0$,

with the convention that $T_{\lambda}=0$ if $\lambda \notin\{0,1,2\}$.

As for pairs, the roles of $T_{0}$ and $T_{2}$ can be exchanged in the above definition, so that, by taking $\tilde{T}_{\alpha}=T_{2-\alpha}$, we obtain a new Peirce grading $T=\tilde{T}_{2} \oplus \tilde{T}_{1} \oplus \tilde{T}_{0}$.

Given a tripotent $e$ in a Jordan triple system $T$ the usual Peirce decomposition of $T=T_{2}(e) \oplus T_{1}(e) \oplus T_{0}(e)$ with respect to $e$ [9 1.1] is an example of Peirce grading of $T$ [9, 1.4].

The formal similarity between Peirce gradings of Jordan pairs and Peirce gradings of triple systems comes from the following obvious though fundamental fact:

2.2. Let $T=T_{2}+T_{1}+T_{0}$ be a decomposition of a Jordan triple system $T$ as a sum of $\Phi$-submodules of $T$. Then $T=T_{2}+T_{1}+T_{0}$ is a Peirce grading of $T$ if and only if $V(T)=V\left(T_{2}\right)+V\left(T_{1}\right)+V\left(T_{0}\right)$ is a Peirce grading of $V(T)$.

2.3. As for pairs, from the multiplication rules given in (2.1) (or from (1.2) with (2.2) ), it is clear that $T_{\alpha}$ is a subtriple of $T$ for $\alpha=0,1,2$ and, moreover, $T_{0}$ and $T_{2}$ are inner ideals of $T$.

2.4. Let $T=T_{2} \oplus T_{1} \oplus T_{0}$ be a Peirce grading of a Jordan triple system $T$ so that $V(T)=V\left(T_{2}\right) \oplus V\left(T_{1}\right) \oplus V\left(T_{0}\right)$ is a Peirce grading of $V(T)$, according to (2.2). Let $I$ be a graded ideal of $V(T)$ which is maximal among all graded ideals of $V(T)$ such that $I^{+} \cap I^{-}=0$. The quotient

$$
V=V(T) / I=\left(T / I^{+}, T / I^{-}\right)
$$

will be called a graded tight double pair of $T$, associated to the grading $T=T_{2} \oplus$ $T_{1} \oplus T_{0}$. By Zorn's lemma, an ideal $I$ satisfying the above conditions does always exist. Moreover, given any graded ideal $L$ of $V(T)$ such that $L^{+} \cap L^{-}=0$, there is an ideal $I$ containing $L$ which is maximal among all graded ideals of $V(T)$ with $I^{+} \cap I^{-}=0$. 
Using (1.5), we have a Peirce grading

$$
V=\left(V\left(T_{2}\right)+I\right) / I \oplus\left(V\left(T_{1}\right)+I\right) / I \oplus\left(V\left(T_{0}\right)+I\right) / I
$$

induced by the grading of $T$.

The behaviour of graded tight double pairs is formally similar to usual tight double pairs [3, 5.1], sometimes replacing ideals by graded ideals. Namely, under the above conditions:

(i) If $0 \neq x \in T$, then either $0 \neq x+I^{+} \in V^{+}$or $0 \neq x+I^{-} \in V^{-}$.

(ii) If $L$ is a nonzero ideal (resp. graded ideal) of $T$, then $\left(\left(L+I^{+}\right) / I^{+}\right.$, $\left.\left(L+I^{-}\right) / I^{-}\right)$is a nonzero ideal (resp. graded ideal) of $V$.

(iii) If $M=\left(M^{+} / I^{+}, M^{-} / I^{-}\right)$is a nonzero graded ideal of $V$, then $M^{+} \cap M^{-}$is a nonzero graded ideal of $T$.

We remark that, unlike (ii), we need in (iii) that the ideal be graded to obtain a nonzero ideal of $T$ since $I$ is maximal only among the graded ideals of $V(T)$ not hitting $T$. However, we are dealing with properties defined in terms of ideals which are not necessarily graded. This difficulty is the motivation of (1.6), which provides the way to obtain graded ideals inside arbitrary ideals.

2.5. Lemma. Let $T=T_{2} \oplus T_{1} \oplus T_{0}$ be a Peirce grading of a Jordan triple system $T$, and let $V=V(T) / I$ be a graded tight double pair of $T$. Then:

(i) $T$ is nondegenerate if and only if $V$ is nondegenerate.

(ii) $T$ is strongly prime if and only if $V$ is strongly prime.

(iii) $T$ is primitive if and only if $V$ is primitive.

(iv) $T$ is simple if and only if $V$ is simple.

Proof. (i) If $V$ is nondegenerate, then $T$ is nondegenerate using (2.4) (i). Conversely, if $T$ is nondegenerate, then $V$ is nondegenerate, as in the proof of 3.4 of [6], where only the fact that $I^{+} \cap I^{-}=0$ is used.

(ii) If $T$ is strongly prime, then $V$ is nondegenerate by (i). Moreover, if $A$ and $B$ are nonzero orthogonal ideals of $V$, then, by (1.6), they contain nonzero orthogonal graded ideals $\tilde{A}$ and $\tilde{B}$ of $V$ which give rise to nonzero orthogonal ideals of $T$ by (2.4) (iii), which is impossible. Conversely, if $V$ is strongly prime, then $T$ is nondegenerate by (i) and it is also prime since nonzero orthogonal ideals of $T$ would give rise to nonzero orthogonal ideals of $V$ by (2.4)(ii).

(iii) If $T$ is primitive, let $K$ be a primitizer of $T$ at $b$ with modulus $c(b, c \in$ $T)$. The proof of $[2,5.8 .2]$ applies here to show that either $K+I^{+} / I^{+} \neq V^{+}$or $K+I^{-} / I^{-} \neq V^{-}$and is a modular inner ideal of $V$. Assume that, for example, $\tilde{K}=K+I^{+} / I^{+} \neq V^{+}$. Now, by (1.6), given any nonzero ideal $A$ of $V$, there exists a nonzero graded ideal $L=\left(L^{+} / I^{+}, L^{-} / I^{-}\right)$of $V$ such that $L \subseteq A$. Thus $L^{+} \cap L^{-}$ is a nonzero ideal of $T$ by (2.4) (iii) so that $K+\left(L^{+} \cap L^{-}\right)=T$ and $\tilde{K}$ complements $L$, hence it also complements $A$. We have shown that $\tilde{K}$ is a primitizer of $V$, hence $V$ is primitive.

Conversely, if, for example, $K / I^{+}$is a primitizer of $V$ at $b+I^{-} \in V^{-}$, then it is readily checked that $K$ is a proper inner ideal of $T$ which is modular at $b$. Moreover, $K$ complements nonzero ideals of $T$ using (2.4)(ii).

(iv) If $T$ is simple, then it is nondegenerate by [5, 2.4 (ii)], hence $V$ is nondegenerate by (i) and, since it is clearly nonzero, it is not trivial. If $A$ is a nonzero ideal of $V$, then there exists a nonzero graded ideal $L=\left(L^{+} / I^{+}, L^{-} / I^{-}\right)$of $V$ such that $L \subseteq A$. Now $L^{+} \cap L^{-}$is a nonzero ideal of $T$ by (2.4)(iii), hence $T=L^{+} \cap L^{-}$, which implies $L=A=V$, and we have shown that $V$ is simple. 
Conversely, assume that $V$ is simple, so that $V$ and hence $T$ are nontrivial. We have that $L=I^{+}+I^{-}$is an ideal of $T$. If $L=0$, then $I=0$ and $V=V(T)$ is simple, which implies $T$ is simple by (0.3) (i) (c). If $L \neq 0$, then $\left(\left(L+I^{+}\right) / I^{+},\left(L+I^{-}\right) / I^{-}\right)=$ $\left(L / I^{+}, L / I^{-}\right)$is a nonzero ideal of $V$. By simplicity of $V, V=\left(L / I^{+}, L / I^{-}\right)=$ $\left(\left(I^{+} \oplus I^{-}\right) / I^{+},\left(I^{+} \oplus I^{-}\right) / I^{-}\right)$and we have $T=I^{+} \oplus I^{-}=T(I)$ is a polarized triple system. Moreover $V=\left(\left(I^{+} \oplus I^{-}\right) / I^{+},\left(I^{+} \oplus I^{-}\right) / I^{-}\right)$is clearly isomorphic to $\left(I^{-}, I^{+}\right)$, hence $\left(I^{-}, I^{+}\right)$is a simple Jordan pair, its opposite $I$ is also simple, and $T=T(I)$ is simple by $(0.3)(\mathrm{ii})(\mathrm{c})$.

2.6. Remark. Notice that, when $T_{1}=T_{0}=0$ and $T_{2}=T$ (which is trivially a Peirce grading of $T$ ), the graded ideals of $V(T)$ are exactly the ideals of $V(T)$, so that a graded tight double pair of $T$ with respect to this grading is a usual tight double pair [3, 5.1]. As a consequence, (2.5) is a generalization of [3, 5.2(ii)] and also yields the equivalence between the simplicity of a Jordan triple system and any of its tight double pairs.

Now we can prove our main result concerning Peirce gradings of Jordan triple systems.

2.7. Theorem. Let $T=T_{2} \oplus T_{1} \oplus T_{0}$ be a Peirce grading of a Jordan triple system T. Let $\alpha \in\{0,2\}$ and assume $T_{\alpha} \neq 0$. Then:

(i) If $T$ is strongly prime (resp. nondegenerate), then $T_{\alpha}$ is strongly prime (resp. nondegenerate).

(ii) If $T$ is primitive (resp. semiprimitive), then $T_{\alpha}$ is primitive (resp. semiprimitive).

(iii) If $T$ is simple, then $T_{\alpha}$ is simple.

Proof. Let $V=V(T) / I$ be a graded tight double pair of $T$ and $V=V_{2} \oplus V_{1} \oplus V_{0}$ be the Pierce grading induced in $V$ by the grading of $T$ as in (2.4). Since $T_{\alpha} \neq 0$, and $I^{+} \cap I^{-}=0$, either $\left(T_{\alpha}+I^{+}\right) / I^{+} \neq 0$ or $\left(T_{\alpha}+I^{-}\right) / I^{-} \neq 0$, i.e., $V_{\alpha} \neq 0$.

(i) If $T$ is nondegenerate, then so is $V(T)$ by (0.3) (i) (a), hence $V\left(T_{\alpha}\right)$ is nondegenerate by (2.2) and [8, 4.1(ii)]. Thus $T_{\alpha}$ is nondegenerate by (0.3) (i) (a).

If $T$ is strongly prime, then $V$ is strongly prime by (2.5) (ii), hence $V_{\alpha}$ is strongly prime by 1.4 (i). If $A, B$ are nonzero orthogonal ideals of $T_{\alpha}$, then $\left(\left(A+I^{+}\right) / I^{+}\right.$, $\left.\left(A+I^{-}\right) / I^{-}\right),\left(\left(B+I^{+}\right) / I^{+},\left(B+I^{-}\right) / I^{-}\right)$are nonzero orthogonal ideals of $V_{\alpha}$, which contradicts primeness of $V_{\alpha}$. We have shown that $T_{\alpha}$ is prime and we already know that it is nondegenerate.

(ii) Notice that a Jordan triple system $T$ is semiprimitive if and only if $V(T)$ is a semiprimitive Jordan pair: if $\operatorname{Rad}()$ denotes the Jacobson radical, $\operatorname{Rad} V(T)=$ $(\operatorname{Rad} T, \operatorname{Rad} T)($ cf. [7. Section 4]). Thus, if $T$ is semiprimitive, then so is $V(T)$, hence $V\left(T_{\alpha}\right)$ is semiprimitive by (2.2) and (1.4)(ii), and $T_{\alpha}$ is semiprimitive.

If $T$ is primitive, then $V$ is primitive by (2.5) (iii), hence $V_{\alpha}$ is primitive by (1.4) (ii). Moreover, $W_{\alpha}=\left(T_{\alpha} /\left(T_{\alpha} \cap I^{+}\right), T_{\alpha} /\left(T_{\alpha} \cap I^{-}\right)\right.$is also primitive since it is isomorphic to $V_{\alpha}$. Let us take, for example, a primitizer $\tilde{K}=K /\left(T_{\alpha} \cap I^{+}\right)$ of $W_{\alpha}$ at $b+\left(T_{\alpha} \cap I^{-}\right) \in W_{\alpha}^{-}\left(b \in T_{\alpha}\right)$. It is very easy to check that $K$ is a proper inner ideal of $T_{\alpha}$ modular at $b$. Moreover, for any nonzero ideal $A$ of $T_{\alpha}$, $\left(\left(A+\left(T_{\alpha} \cap I^{+}\right)\right) /\left(T_{\alpha} \cap I^{+}\right),\left(A+\left(T_{\alpha} \cap I^{-}\right)\right) /\left(T_{\alpha} \cap I^{-}\right)\right)$is a nonzero ideal of $W_{\alpha}$, hence $\tilde{K}+\left(A+\left(T_{\alpha} \cap I^{+}\right)\right) /\left(T_{\alpha} \cap I^{+}\right)=W_{\alpha}$, and $K+A=T_{\alpha}$, i.e., $K$ is a primitizer for $T_{\alpha}$. 
(iii) If $T$ is simple, then $V$ is simple by (2.5) (iv), hence $V_{\alpha}$ is simple by (1.4) (iii). As above, $W_{\alpha}=\left(T_{\alpha} /\left(T_{\alpha} \cap I^{+}\right), T_{\alpha} /\left(T_{\alpha} \cap I^{-}\right)\right.$is isomorphic to $V_{\alpha}$, hence $W_{\alpha}$ is simple. Now, since $M=I \cap V\left(T_{\alpha}\right)=\left(T_{\alpha} \cap I^{+}, T_{\alpha} \cap I^{-}\right)$is an ideal of $V\left(T_{\alpha}\right)$, $\left(T_{\alpha} \cap I^{+}\right)+\left(T_{\alpha} \cap I^{-}\right)$is an ideal of $T_{\alpha}$ and

$$
L=\left(\left[\left(T_{\alpha} \cap I^{+}\right)+\left(T_{\alpha} \cap I^{-}\right)\right] /\left(T_{\alpha} \cap I^{+}\right),\left[\left(T_{\alpha} \cap I^{+}\right)+\left(T_{\alpha} \cap I^{-}\right)\right] /\left(T_{\alpha} \cap I^{-}\right)\right)
$$

is an ideal of $W_{\alpha}$. By simplicity of $W_{\alpha}$, either $L=0$ or $L=W_{\alpha}$.

If $L=0$, then $T_{\alpha} \cap I^{+}=T_{\alpha} \cap I^{-}=0, W_{\alpha}$ is isomorphic to $V\left(T_{\alpha}\right)$, which is then simple, and $T_{\alpha}$ is simple by $(0.3)(\mathrm{i})(\mathrm{c})$.

If $L=W_{\alpha}$, then $W_{\alpha}$ is isomorphic to $\left(T_{\alpha} \cap I^{-}, T_{\alpha} \cap I^{+}\right)$, hence $\left(T_{\alpha} \cap I^{-}, T_{\alpha} \cap I^{+}\right)$ and its opposite $M$ are simple. Moreover, we also have that

$$
T_{\alpha}=\left(T_{\alpha} \cap I^{+}\right)+\left(T_{\alpha} \cap I^{-}\right)=\left(T_{\alpha} \cap I^{+}\right) \oplus\left(T_{\alpha} \cap I^{-}\right)=T(M),
$$

and $T_{\alpha}$ is simple by (0.3)(ii)(c).

2.8. Remark. We will not explicitly deal with Jordan algebras since every reasonable definition of Peirce grading for algebras (formally reproducing the multiplication rules of Peirce decompositions with respect to idempotents) would be in particular a Peirce grading of the underlying triple system, giving rise, by duplication, to a Peirce grading of the corresponding double pair. Since all regularity conditions we have considered are equivalent for a Jordan algebra $J$ and the pair $V(J)$ (nondegeneracy with obvious proof, semiprimitivity by [7, 4.17], strong primeness by [6] 1.12], primitivity by [4, 0.11], and simplicity by [5, 3.4]) they are inherited by the diagonal components of a Peirce grading of $J$, applying [8, 4.1(ii)] and (1.4) to $V(J)$.

\section{ACKNOWLEDGMENTS}

We thank Professor Ottmar Loos for allowing us to include his result (Lemma 1.3) in our paper, as well as for bringing the problem of the inheritance of regularity by the components of Peirce gradings to our attention.

\section{REFERENCES}

[1] A. D'Amour, "Quadratic Jordan Systems of Hermitian Type". J. Algebra 149 (1992), 197233. MR 93d:17041

[2] A. D'Amour, K. McCrimmon, "The Local Algebras of Jordan Systems". J. Algebra 177 (1995), 199-239. MR 96k:17047

[3] J. A. Anquela, T. Cortés, "Primitive Jordan Pairs and Triple Systems". J. Algebra 184 (1996), 632-678. MR 98b:17033

[4] J. A. Anquela, T. Cortés, "Primitivity in Jordan Systems is Ubiquitous". J. Algebra 202 (1998), 295-314. MR 99a:17030

[5] J. A. Anquela, T. Cortés, "Local and Subquotient Inheritance of Simplicity in Jordan Systems". J. Algebra 240 (2001), 680-704.

[6] J. A. Anquela, T. Cortés, O. Loos, K. McCrimmon, "An Elemental Characterization of Strong Primeness in Jordan Systems". J. Pure Appl. Algebra 109 (1996), 23-36. MR 97j:17035

[7] O. Loos, Jordan Pairs, Lecture Notes in Mathematics, Vol. 460, Springer-Verlag, New York, 1975. MR 56:3071]

[8] O. Loos, E. Neher, "Complementation of Inner Ideals in Jordan Pairs". J. Algebra 166 (1994), 255-295. MR 95f:17028

[9] K. McCrimmon, "Peirce Ideals in Jordan Triple Systems". Pac. J. Math. 83 (2) (1979), 415-439. MR 81i:17012 
[10] K. McCrimmon, E. Zelmanov, "The Structure of Strongly Prime Quadratic Jordan Algebras". Adv. Math. 69 (2) (1988), 133-222. MR 89k:17052

[11] E. Neher "Involutive Gradings in Jordan Structures". Comm. Algebra 9 (6) (1981), 575-599. MR 82i:17025

Departamento de Matemáticas, Universidad de Oviedo, C/ Calvo Sotelo s/n, 33007 Oviedo, Spain

E-mail address: anque@pinon.ccu.uniovi.es

Departamento de Matemáticas, Universidad de Oviedo, C/ Calvo Sotelo s/n, 33007 OVIEDO, Spain

E-mail address: cortes@pinon.ccu.uniovi.es 